\title{
Physical modeling of untrenched submarine pipeline instability
}

\author{
F.P. Gao ${ }^{\mathrm{a}, \mathrm{b}}$, X.Y. Gu ${ }^{\mathrm{b}}$, D.S. Jeng ${ }^{\mathrm{c}, *}$ \\ ${ }^{a}$ Special Research Centre for Offshore Foundation System, The University of Western Australia, \\ Nedlands, WA 6907, Australia \\ ${ }^{\mathrm{b}}$ Institute of Mechanics, Chinese Academy of Sciences, Beijing 100080, China \\ c School of Engineering, Gold Coast Campus, Griffith University, Gold Coast, QLD 9726, Australia
}

Received 8 April 2002; accepted 17 July 2002

\begin{abstract}
Wave-induced instability of untrenched pipeline on sandy seabed is a 'wave-soil-pipeline' coupling dynamic problem. To explore the mechanism of the pipeline instability, the hydrodynamic loading with U-shaped oscillatory flow tunnel is adopted, which is quite different from the previous experiment system. Based on dimensional analysis, the critical conditions for pipeline instability are investigated by altering pipeline submerged weight, diameter, soil parameters, etc. Based on the experimental results, different linear relationships between Froude number $(F r)$ and non-dimensional pipeline weight $(G)$ are obtained for two constraint conditions. Moreover, the effects of loading history on the pipeline stability are also studied. Unlike previous experiments, sand scouring during the process of pipe's losing stability is detected in the present experiments. In addition, the experiment results are compared with the previous experiments, based on Wake II model for the calculation of wave-induced forces upon pipeline. It shows that the results of two kinds of experiments are comparable, but the present experiments provide better physical insight of the wave-soil-pipeline coupling effects. (C) 2002 Elsevier Science Ltd. All rights reserved.
\end{abstract}

Keywords: Pipeline instability; Oscillatory flow; Wave loading; Wake II model; Wave-soil-pipeline coupling

* Corresponding author. Tel.: +61-7-5552-8683; fax: +61-7-5552-8065.

E-mail address: d.jeng@mailbox.gu.edu.au (D.S. Jeng). 


\section{Introduction}

Submarine pipelines are a convenient means to transport natural oil or gas from offshore oil wells to an onshore location. One of the main problems encountered with the use of the pipeline is the wave-induced instability (Herbich, 1985). Under the wave loading, there exists a balance between wave forces, submerged weight of pipelines and soil resistance. To avoid swept sideways, the pipeline ought to be given a heavy enough concrete coating, or it has to be anchored or trenched. However, both designs are expensive and complicated. Thus, a better understanding of the wave-induced untrenched pipeline stability is important for pipeline design.

The interaction between ocean waves, submarine pipeline and seabed has attracted more and more attention over the past few decades. Coulomb friction theory was employed to estimate the friction force between pipeline and soil, under the action of ocean waves before 1970s. Actually, Coulomb friction theory is far from the realistic wave-induced pipe-soil interaction. Lyons (1973) experimentally explored the wave-induced stability of untrenched pipeline, and concluded that the Coulomb friction theory was not suitable to describe the wave-induced interaction between pipeline and soil, especially when adhesive clay is involved. This is because that the lateral friction between pipeline and soil should be the function of properties of soil, pipe and wave.

Two large model test programs have been conducted by SINTEF in 1980s, in which the pipeline-seabed interaction was examined with full diameter pipe segments (see Fig. 1). These are the multi-client project 'PIPESTAB' (1985-1987) and the 'AGA-project' (1987-1988) (Allen et al., 1989; Brennodden et al., 1986; Wagner et al., 1987; Brennodden et al., 1989). A considerable experience was gained, including an empirical pipe-soil interaction model and an energy based pipe-soil interaction model proposed by Wagner et al. (1987) and Brennodden et al. (1989), respect-

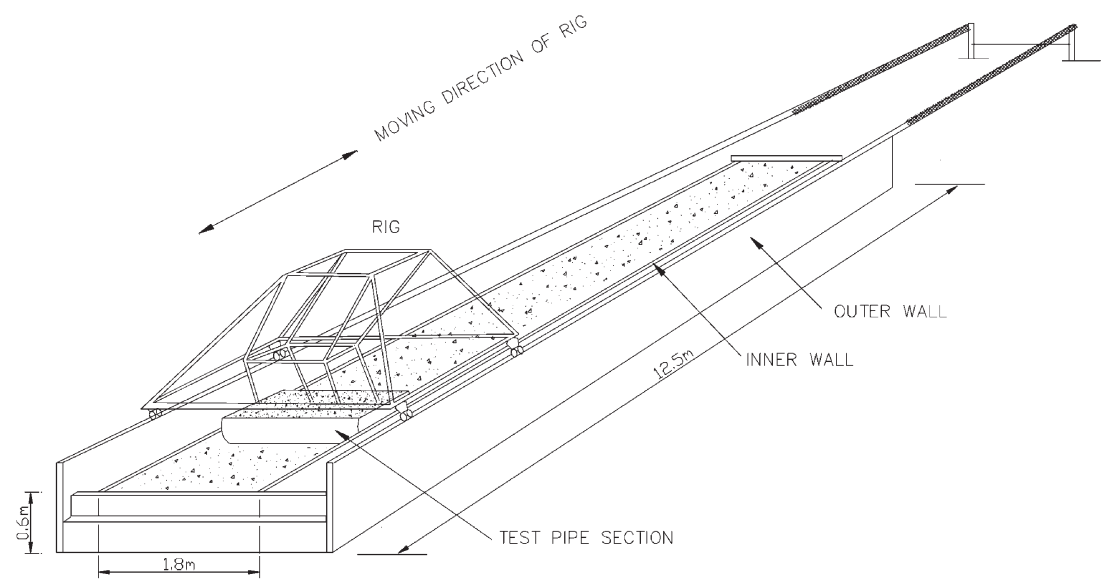

Fig. 1. Typical test facility for pipe-soil interaction study by SINTEF (adapted from Wagner et al., 1987). 
ively. In both models, the total lateral resistance $F_{\mathrm{H}}$, was assumed as the sum of sliding resistance component $F_{\mathrm{F}}$ and soil passive resistance component $F_{\mathrm{R}}$, i.e.

$$
F_{\mathrm{H}}=F_{\mathrm{F}}+F_{\mathrm{R}}
$$

where

$$
F_{\mathrm{F}}=\mu\left(W_{\mathrm{s}}-F_{\mathrm{L}}\right),
$$

where, $\mu$ is the sliding resistance coefficient, $W_{\mathrm{s}}$ is the pipeline submerged weight per meter, and $F_{\mathrm{L}}$ is the wave-induced lift force upon pipeline. The difference between two models is the methods for calculating the soil resistance component. In the former model (the empirical pipe-soil interaction model, Wagner et al., 1987)

$$
F_{\mathrm{R}}=\beta \gamma^{\prime} A_{\mathrm{T}},
$$

where $\beta$ is an empirical coefficient, $\gamma^{\prime}$ the soil buoyant weight, and $A_{\mathrm{T}}$ is half of the contact area between pipeline and soil. However, in the latter model (energy based pipe-soil interaction, Brennodden et al., 1989), $F_{\mathrm{R}}$ is relative to the work done by pipe during its movement. These experimental results and the models deduced from the results form an important basis for today's regulations regarding pipeline stability design (Det norske Veritas, 1988).

In the above experiments, the cyclic loadings are exerted with mechanical actuators to simulate the real wave-induced forces upon pipeline (see Fig. 1). Moreover, the pressure upon seabed could not be simulated in their experiments. These pressure fluctuations further induce the variations in effective stresses and pore water pressure within non-cohesive marine sediments. They are different from the actual hydrodynamic wave situations. In reality, the hydrodynamic forces act not only on pipeline but also seabed, and the response of seabed to the hydrodynamic forces can directly affect the pipeline stability. Therefore, precisely speaking, the wave-induced on-bottom stability of the submarine pipeline involves the interaction of wave, soil and pipe, not only pipe-soil interaction. Additionally, in the above pipe-soil interaction models (Wagner et al., 1987; Brennodden et al., 1989), numerous empirical coefficients have no implicit physical meanings and are difficult to be determined in design procedure. To date, it seems that the underlying physical mechanism is not yet well understood, as stated by Hale et al. (1991).

Regarding the interaction between waves, pipes and sandy seabeds, many investigations have been conducted in the study on sand scouring near pipelines (Sumer and Fredsoe, 1991; Chiew, 1990; Mao, 1988). In the aforementioned experimental approaches, the pipeline was installed at the fixed condition, thus, the pipeline instability was not involved.

This paper aims to explore the wave-induced instability of untrenched pipeline with hydrodynamic experiments through physical modeling. Based on Wake II model, the wave-induced forces on pipeline are calculated. With this, the present experiment results are compared with that of previous pipe-soil interaction experiments. 


\section{Experimental set-up}

\subsection{Experimental facilities and instruments}

Under the wave action, the water particles oscillate elliptically at upper water level with certain frequency. But due to the boundary effect, the particles near the sea bottom mainly oscillate horizontally, which directly affect the pipeline stability. To simulate the oscillating movement of water particles near the seabed, experiments are conducted in the U-shaped oscillatory flow water tunnel, as shown in Fig. 2.

The water tunnel is made of apparent plexiglass with section area of $0.2 \times 0.2 \mathrm{~m}^{2}$. By a butterfly valve, periodically opening and closing at the top of a limb of the water tunnel, the water accomplishes a simple harmonic oscillation

$$
A=A_{0}(t) \sin \omega t,
$$

where $A_{0}(t)$ is the amplitude of oscillatory flow; $\omega$ the angle velocity of oscillatory flow, i.e. $\omega=2 \pi / T ; T$ the period of oscillatory flow, $T=2.60 \mathrm{~s}$; and $t$ is the loading time. By regulating the valve, the effective air flux from air blower can be changed. Thus, the amplitude can be varied continuously within 5-200 mm.

The lower part of the water tunnel constitutes the test section, under which a soil box with length of $0.60 \mathrm{~m}$, width of $0.20 \mathrm{~m}$, depth of $0.035 \mathrm{~m}$ is constructed. The soil box is filled with sand, which is regarded as sand bed at the sea bottom.

The test pipe is directly laid upon the surface of sand, as shown in Fig. 3. As to a long distance laid pipeline, the stability of pipeline at separate sections is different. For example, the demand for the stability of pipeline sections near risers is higher than normal sections. In the actual pipeline design, different safety factors are chosen. Due to the constraints from risers and pipeline's anti-torsion rigidity, the movement of the pipeline is not purely horizontal or rotational. Thus, the following two constraint conditions are considered:

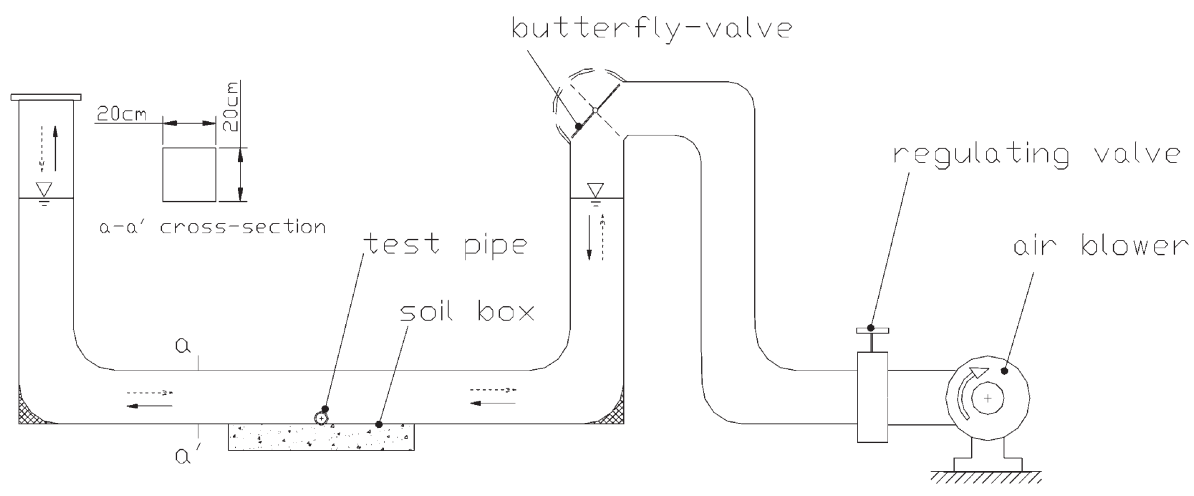

Fig. 2. The sketch of U-shaped oscillatory water flow tunnel. 


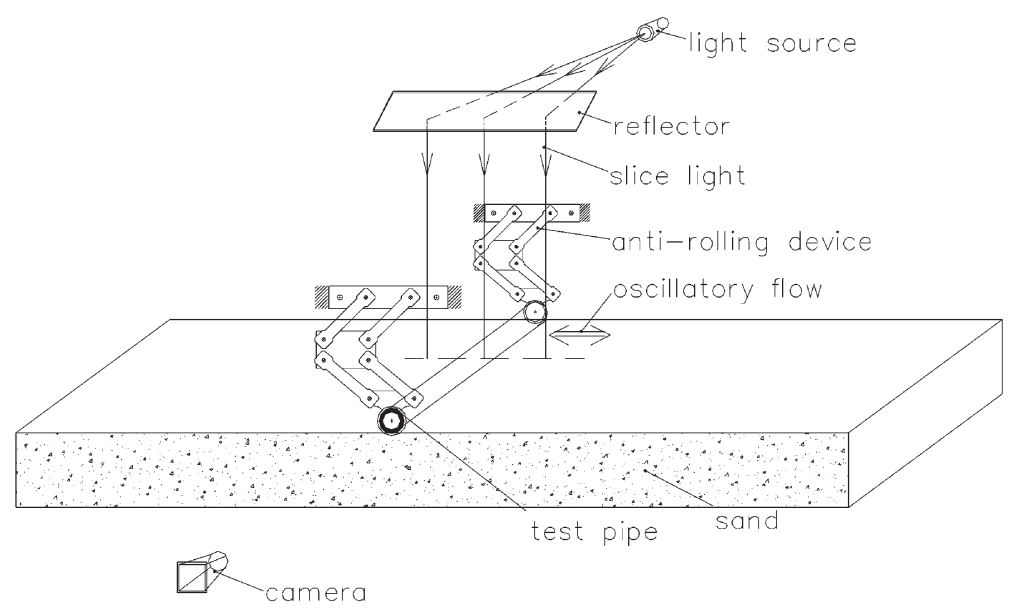

Fig. 3. Schematic diagram of testing method.

Case I: Pipeline is free at its ends;

Case II: Pipeline's rolling is restricted, but pipeline can move freely in horizontal and vertical directions.

For this purpose, a device for anti-rolling of pipeline was designed (see Fig. 3). The anti-rolling device is made of thin plexiglass plate and mini bearings. It includes two parts, which are installed at the two ends of pipeline separately.

To detect the onset of sand scouring, sand scour visualization was carried out under the sliced light by a video camera. Meanwhile, the instability process of the pipe was also observed and recorded by the video camera, as shown in Fig. 3.

\subsection{Froude modeling}

Development and testing of offshore pipeline model are of great importance because of the difficulty of obtaining data from prototypes. However, care must be taken to make sure that the model simulates the behavior of the prototype as accurately as possible.

In the study of wave-pipeline interaction problem, three non-dimensional numbers relative to flow characteristics can be deduced. They are:

(a) Froude number $\mathrm{Fr}$

$$
F r=\frac{U_{\mathrm{m}}}{(g D)^{1 / 2}}
$$

which is the ratio of inertia force to gravitational force, which reflects the dynamic similarity of flow with gravity forces acting;

(b) Keulegan-Carpenter number, KC 


$$
\mathrm{KC}=\frac{U_{\mathrm{m}} T}{D}
$$

which controls the generation and development of vortex around pipeline, and is related to the hydrodynamic force on the pipe under wave loading;

(c) Reynolds number, $R e$

$$
R e=\frac{U_{\mathrm{m}} D}{v},
$$

which is the ratio of inertia force to viscous force.

In the case of ocean wave with a free surface, the gravitational effect predominates, and pipeline on-bottom stability is relative to pipeline's submerged weight. The effect of other factors, such as viscosity, surface tension, etc. is generally small and can be neglected. Since both $F r$ and $R e$ cannot be satisfied concurrently during model tests, it is convenient to employ the Froude scaling process and allowance is made for variation in Reynolds number (Chakrabarti, 1994).

According to Froude's law, the following scales should be maintained:

$$
\frac{\lambda_{U_{\mathrm{m}}}}{\lambda_{\mathrm{g}}^{1 / 2} \lambda_{D}^{1 / 2}}=1,
$$

where $\gamma$ represents the ratio of the parameters of model to that of prototype.

From Eq. (8), since $\lambda_{\mathrm{g}}=1$, we have

$$
\begin{aligned}
& \lambda_{U_{\mathrm{m}}}=\lambda_{D}^{1 / 2}, \\
& \lambda_{\mathrm{T}}=\frac{\lambda_{D}}{\lambda_{U_{\mathrm{m}}}}=\lambda_{D}^{1 / 2} .
\end{aligned}
$$

Therefore,

$$
\lambda_{\mathrm{KC}}=\frac{\lambda_{U_{\mathrm{m}}} \lambda_{\mathrm{T}}}{\lambda_{D}}=1 .
$$

This indicates that $F r$ and $\mathrm{KC}$ can be satisfied concurrently during the model tests. Because the Keulegan-Carpenter number follows Froude's law, dependence on KC ensures that the model values are applicable to prototype. However, if the quantities strongly depend on Reynolds number, direct scaling is not possible.

Sandy bottom is distributed in many areas in South China Sea, where Fr and KC change between $0-0.5$ and $0-20$, respectively. In our experiments, the values of $F r$ and $\mathrm{KC}$ vary within these ranges. The Reynolds number is smaller than the actual value by two orders. 


\subsection{Testing materials}

\subsubsection{Soils}

Because of the proximity of the pipeline to the seafloor, the modeling of soil characteristic of the foundation may be important. The sand beds consist of medium sand and fine sand. The index properties of the sands are shown in Table 1. The moist sand is first saturated, then packed in the soil box under water, and finally trimmed with a scraper. The difference of the unit weights for different tests is controlled within the error of $5 \%$.

\subsubsection{Pipelines}

The pipe model spans the soil surface vertically to the direction of oscillatory flow, as shown in Fig. 3. The length of the pipe model should be sufficient to minimize the ending effects. To simulate the two dimensional problem, the pipe ends are close to the vertical walls of U-shaped tunnel. In the experiments, the gaps between pipe ends and the U-shaped tunnel walls are about $5 \mathrm{~mm}$. Thus, scouring at the end of the pipe model is not considered a problem, which has been proved in the tests.

The submarine pipeline generally has a large span so that the pipeline model may be treated as a two-dimensional structure. The submerged weight of pipeline directly determines the contact force between pipeline and seabed, and further affects onbottom stability around the pipeline.

The weight of the pipe is adjusted to model the typical submerged weight of actual pipeline, according to the similarity parameter $G$, i.e.

$$
G=\frac{W_{\mathrm{s}}}{\gamma^{\prime} D^{2}},
$$

where, $\gamma^{\prime}$ is buoyant unit weight of soil, $\gamma^{\prime}=\left(\rho_{\text {sat }}-\rho_{\mathrm{w}}\right) g$. That is, the model and prototype can be expressed by

$$
G=\frac{\left(W_{\mathrm{s}}\right)_{\mathrm{p}}}{\gamma_{\mathrm{p}}^{\prime} D_{\mathrm{p}}^{2}}=\frac{\left(W_{\mathrm{s}}\right)_{\mathrm{m}}}{\gamma_{\mathrm{m}}^{\prime} D_{\mathrm{m}}^{2}},
$$

where the subscripts $p$ and $m$ stand for prototype and model, respectively. The testing

Table 1

\begin{tabular}{|c|c|c|c|c|c|c|}
\hline $\begin{array}{l}\text { Mean grain } \\
\text { size, } d_{50} \\
(\mathrm{~mm})\end{array}$ & $\begin{array}{l}\text { Grain size at } \\
\text { which } 10 \% \\
\text { of the soil } \\
\text { weight is } \\
\text { finer, } d_{10} \\
(\mathrm{~mm})\end{array}$ & $\begin{array}{l}\text { Uniformity } \\
\text { coefficient, } C_{\mathrm{u}}\end{array}$ & $\begin{array}{l}\text { Unit weight, } \\
\gamma\left(\mathrm{kN} / \mathrm{m}^{3}\right)\end{array}$ & $\begin{array}{l}\text { Dry unit } \\
\text { weight, } \\
\gamma_{\mathrm{d}}\left(\mathrm{kN} / \mathrm{m}^{3}\right)\end{array}$ & $\begin{array}{l}\text { Initial void } \\
\text { ratio, } e_{0}\end{array}$ & $\begin{array}{l}\text { Relative } \\
\text { density, } D_{\mathrm{r}}\end{array}$ \\
\hline 0.38 & 0.30 & 1.4 & 19.00 & 14.80 & 0.73 & 0.37 \\
\hline 0.21 & 0.11 & 2.0 & 21.05 & 17.47 & 0.56 & 0.60 \\
\hline
\end{tabular}

Index properties of the test sands 
pipes are composed of aluminum, with length of $0.19 \mathrm{~m}$. The pipes are divided into three groups with different diameters: $0.014,0.020$ and $0.030 \mathrm{~m}$. In each group, pipes have different weights. The diameter $D$ and submerged weight $W_{\mathrm{s}}$ of test pipes are listed in Table 2.

According to dimensional analysis, Eq. (13) can also be expressed as

$$
\frac{\lambda_{W_{\mathrm{s}}}}{\lambda_{\gamma} \lambda_{D}^{2}}=1
$$

When $\lambda_{\gamma^{\prime}}=1$, then the ratio of the pipeline submerged weight of model to that of prototype is

$$
\lambda_{W_{\mathrm{s}}}=\lambda_{D}^{2}
$$

Due to the pipe weight and the operation reason, some initial embedment always does exist, although the amount of embedment is very small. Conventionally, $e / D=0.03-0.05$, where $e$ is pipe initial embedment.

\subsection{Testing procedures}

To explore the mechanism of pipeline instability induced by rapidly increasing storm wave, a constant velocity of oscillatory flow amplitude $\dot{A}_{0}, \dot{A}_{0}=$ $9 \times 10^{-3} \mathrm{~cm} / \mathrm{s}$, was adopted first in the experiments.

From Eq. (4), the velocity of oscillatory flow can be deduced

$$
U(t)=\dot{A}_{0}(t) \sin \omega t+\omega A_{0} \cos \omega t .
$$

\begin{tabular}{|c|c|c|c|c|c|c|c|}
\hline \multicolumn{6}{|l|}{ Case I } & \multicolumn{2}{|l|}{ Case II } \\
\hline $\begin{array}{l}\text { Pipe } \\
\text { diameter, } \\
D(\mathrm{~m})\end{array}$ & $\begin{array}{l}\text { Submerged } \\
\text { weight, } W_{\mathrm{s}} \\
(\mathrm{N} / \mathrm{m})\end{array}$ & $\begin{array}{l}\text { Pipe } \\
\text { diameter, } \\
D(\mathrm{~m})\end{array}$ & $\begin{array}{l}\text { Submerged } \\
\text { weight, } W_{\mathrm{s}} \\
(\mathrm{N} / \mathrm{m})\end{array}$ & $\begin{array}{l}\text { Pipe } \\
\text { diameter, } \\
D(\mathrm{~m})\end{array}$ & $\begin{array}{l}\text { Submerged } \\
\text { weight, } W_{\mathrm{s}} \\
(\mathrm{N} / \mathrm{m})\end{array}$ & $\begin{array}{l}\text { Pipe } \\
\text { diameter, } \\
D(\mathrm{~m})\end{array}$ & $\begin{array}{l}\text { Submerged } \\
\text { weight, } W_{\mathrm{s}} \\
(\mathrm{N} / \mathrm{m})\end{array}$ \\
\hline 0.030 & 1.52 & 0.020 & 1.09 & $0.030 *$ & 1.61 & 0.030 & 1.51 \\
\hline 0.030 & 2.00 & 0.020 & 1.35 & $0.030 *$ & 2.00 & 0.030 & 2.04 \\
\hline 0.030 & 2.40 & 0.020 & 1.54 & $0.030 *$ & 2.40 & 0.030 & 2.59 \\
\hline 0.030 & 3.12 & 0.020 & 1.72 & $0.030 *$ & 3.12 & 0.030 & 2.94 \\
\hline 0.030 & 3.53 & 0.020 & 1.97 & $0.030 *$ & 3.53 & 0.020 & 0.78 \\
\hline 0.030 & 3.93 & 0.014 & 0.78 & $0.030 *$ & 3.93 & 0.020 & 0.98 \\
\hline 0.030 & 4.22 & 0.014 & 0.89 & $0.030 *$ & 4.22 & 0.020 & 1.12 \\
\hline 0.030 & 4.40 & 0.014 & 1.05 & $0.030 *$ & 4.50 & 0.020 & 1.29 \\
\hline 0.030 & 5.00 & 0.014 & 1.21 & $0.030 *$ & 5.00 & & \\
\hline 0.030 & 5.24 & & & $0.030 *$ & 5.29 & & \\
\hline
\end{tabular}

Table 2

The parameters of test pipes

*Fine sand; others-medium sand. 
(a)

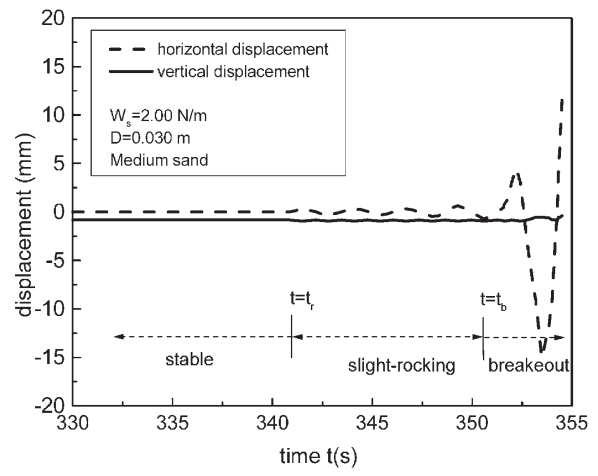

(b)

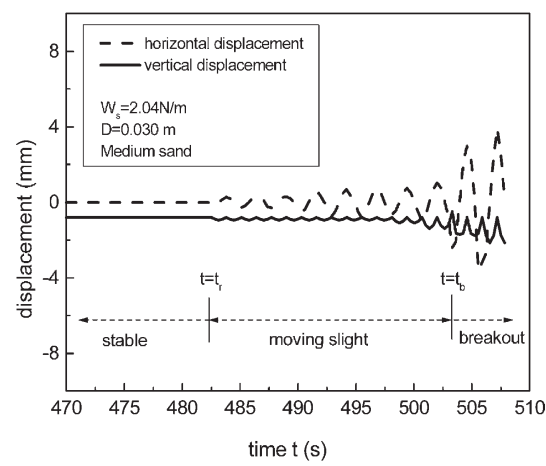

Fig. 4. Pipe displacement-time curves.

In the experiments, $A_{0}(t)$ is in the order of $10^{-1}(\mathrm{~m})$, thus $\dot{A}_{0} / \omega A_{0}=0\left(10^{-3}\right)$. Therefore, the maximum water particle velocity of the oscillating flow $U_{\mathrm{m}}$ is as follows:

$$
U_{\mathrm{m}}=\omega A_{0}(t) \text {. }
$$

In other words, the maximum water particle velocity is mainly relative to the angle velocity and the current flow amplitude.

Furthermore, since the storm growing is not always continuous, it is necessary to examine the effects of loading history on pipeline instability, which will be described in Section 3.3.

During the experiments, the water level change was recorded with a water differential pressure transducer and data acquisition system.

\section{Experiment results}

\subsection{Pipeline instability process}

When the amplitude of oscillatory flow $A_{0}$ increases continuously with a constant velocity, the pipeline displacements are recorded (see Fig. 4). The following three characteristic times can be identified in the pipeline instability process:

1. $t=t_{\mathrm{s}}$ : At a certain distance apart from the pipe, the sand grains at the bed surface start to move visibly. Onset of scour occurs (see Fig. 5). When the water particle

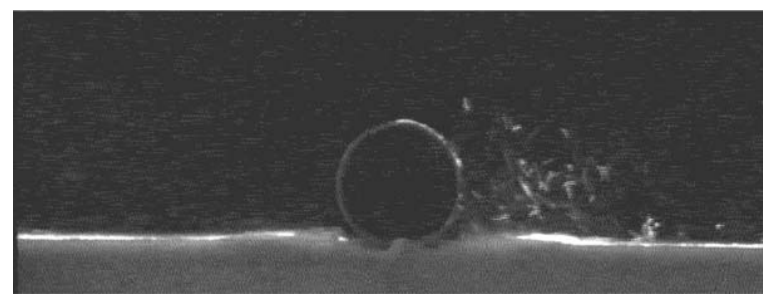

Fig. 5. Onset of sand scouring. 
velocity is large enough to make considerable amount of sediment into suspension, sand ripples are gradually formed in the vicinity of the pipe.

2. $t=t_{\mathrm{r}}$ : The pipe begins to move slightly (see Figs. 4 and 6). As to Case I, the pipe mainly swings at its original site, and its vertical settlement is nearly invisible. But for Case II, both vertical and horizontal movements develop gradually. The horizontal displacement is about $1-3 \% D$, while the vertical settlement is approximately less than $1 \% D$.

3. $t=t_{\mathrm{b}}$ : Pipe breakout takes place at a short time (see Figs. 4 and 6). As to Case $\mathrm{I}$, the pipe begins detaching from its original place for few cycles. Then it rolls away immediately, and sometimes it rolls over the sand ripple nearby. But, as for Case II, the pipe pushes the sand aside with the horizontal displacement of approximately $20-30 \% D$.

The pipeline instability is always coupled with sand scouring. However, as to the pipelines whose submerged weights are small, the pipeline breaks out when the oscillatory flow is not strong enough to induce sand scour.

\subsection{Criterion for pipeline on-bottom instability}

The wave-induced instability of pipelines with two constraint conditions, i.e. Case I and Case II, was studied, respectively.

Case I: Freely laid pipeline

In order to explore the effects of sand properties on pipeline instability, the experiments on the instability of pipelines with various diameters and weights were conducted on medium sand and fine sand separately, whose properties are listed in Table 1. The oscillatory flow amplitudes at which the pipe loses stability $\left(A_{0}=A_{\mathrm{b}}\right)$ were recorded. With Eqs. (5), (6) and (17), KC and Fr numbers can be obtained by

$$
\begin{aligned}
& \mathrm{KC}=\frac{2 \pi A_{\mathrm{b}}}{D}, \\
& F r=\frac{2 \pi A_{\mathrm{b}}}{T(g D)^{1 / 2}} .
\end{aligned}
$$

Fig. 7 shows the correlation between $G$ and KC numbers. As to the pipelines with same diameter, $\mathrm{KC}$ at which pipelines lose stability increases linearly with $G$ number. But the relationships are different for different diameters. It shows that when using $\mathrm{KC}$ number for data reduction, pipeline diameter effect is significant.

Fig. 8 shows the correlation between $F r$ and $G$. For the same sand (medium sand),
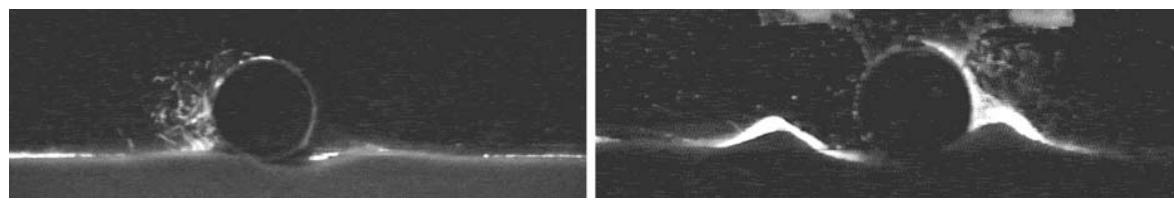

Fig. 6. Phenomena of pipeline losing stability. 


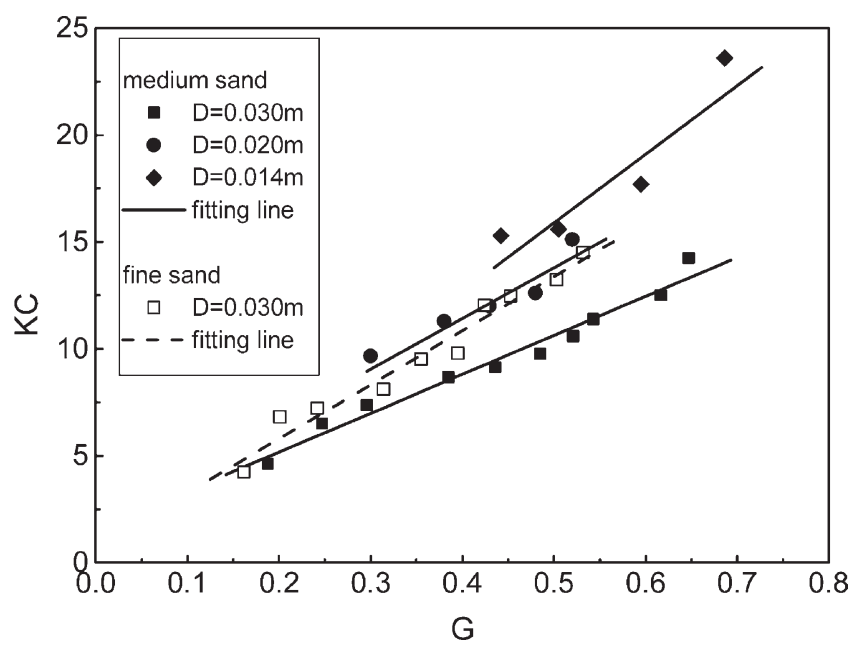

Fig. 7. $\mathrm{KC}$ and $G$ correlation (Case I).

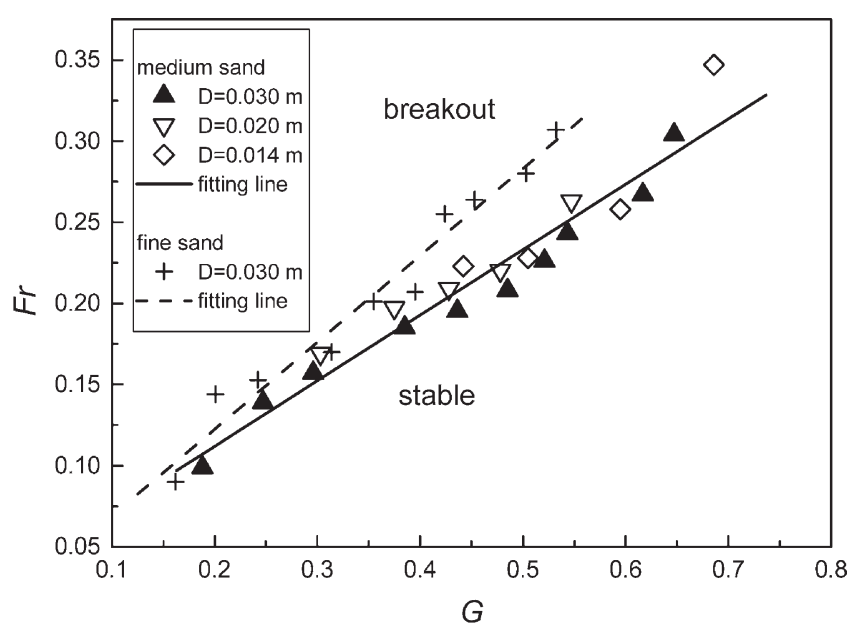

Fig. 8. $F r$ and $G$ correlation for medium sand (Case I).

all the data with different pipe diameters fall within the range with the same linear relationship. There is a good correlation between the $F r$ number and the $G$ number regardless of the pipeline diameter. It matches with the point of Chakrabarti (1994) and Poorooshasb (1990), which indicates the importance of $F r$ in case of waterstructure-soil interaction. However, there exists some difference between the results for medium sand and fine sand, as shown in Figs. 7 and 8. That is, the sand characteristics influence pipeline stability.

Case II: Anti-rolling pipeline

With the designed anti-rolling device (Fig. 3), experiments were conducted on 


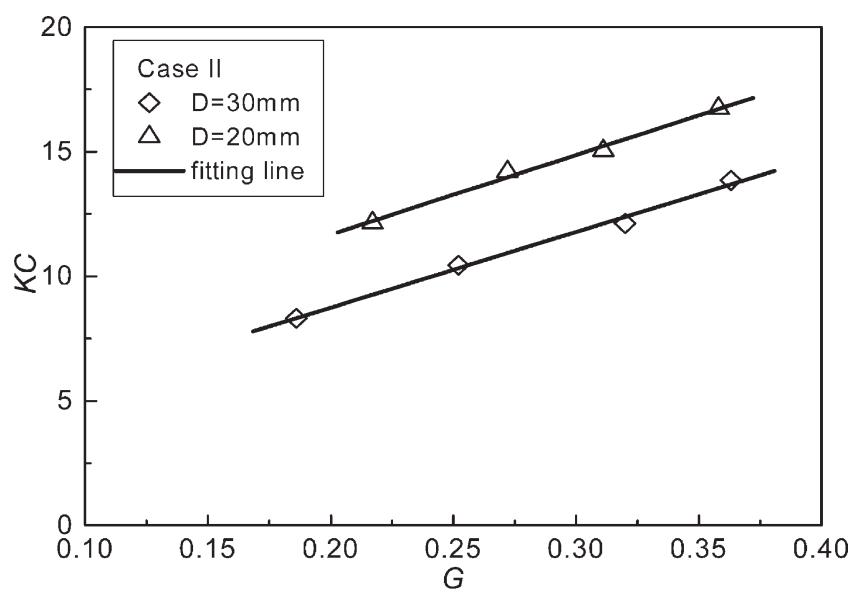

Fig. 9. KC and $G$ correlation (Case II).

medium sand for pipelines with different diameters, i.e. $D=0.030,0.020 \mathrm{~m}$, as well as different submerged weight. As the experiments on pipelines were freely laid, the oscillatory flow amplitude also rises at the same speed $\dot{A}_{0}$.

Fig. 9 shows the correlation between $G$ and KC numbers for Case II. Similar to Case I, for the pipelines with same diameter, $\mathrm{KC}$ at which pipelines lose stability increases linearly with $G$ number, but pipeline diameter effect is also very obvious.

Fig. 10 shows the correlation between $F r$ and $G$ for Case II. All the data with different pipe diameters fall within the range with the same linear relationship, as Case I shown in Fig. 10.

Herein, it should be mentioned that the experiment results are obtained under the

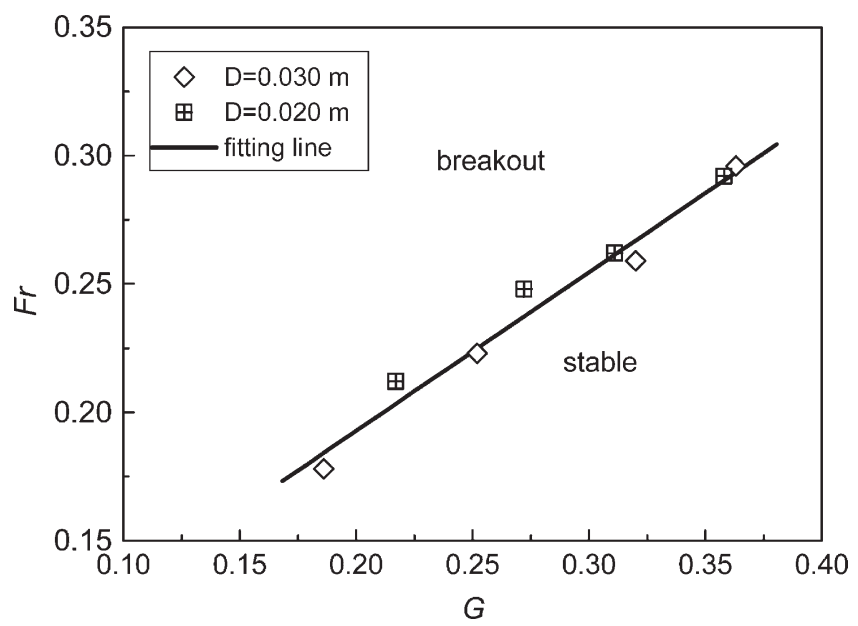

Fig. 10. $F r$ and $G$ correlation (Case II). 
condition of the oscillatory flow amplitude rising at the same speed $\dot{A}_{0}$. As to the pipelines with different submerged weights, the $F r$ number at which pipelines lose stability are different. Thus, the oscillating time $(t / T)$ is different in different experiments. Their ranges for Case I and Case II are about 80-300, 170-230, respectively. Under the oscillating actions, the effective stress field and pore-pressure field will change with time. When the oscillatory flow velocity exceeds a certain value, the sand beside pipeline is scoured under the influence of vortexes. The sand ripples are formed step by step. They will affect the flow field near pipeline and even influence pipeline instability.

In addition, the $F r-G$ relationships for the two constrains are obtained within the certain range of non-dimensional parameter $G$. As mentioned, $\mathrm{KC}$ can be satisfied concurrently with $\mathrm{Fr}$ during model tests. The $\mathrm{KC}$ range in the experiments is about $5-20$.

\subsection{Effects of loading history}

In the aforementioned experiments, the same wave loading method was adopted to examine the instability induced by rapidly increasing storm. However, real storm wave events are unpredictable and the field conditions are often characterized with significant uncertainty. Thus, it is also very necessary to study the effects of loading history upon the pipeline instability.

\subsubsection{Effects of loading velocity}

First, various loading velocities are employed, i.e. (a) $\dot{A}_{0}=4.5 \times 10^{-3} \mathrm{~cm} / \mathrm{s}$, (b) $\dot{A}_{0}=9.0 \times 10^{-3} \mathrm{~cm} / \mathrm{s}$, and (c) $\dot{A}_{0}=1.8 \times 10^{-2} \mathrm{~cm} / \mathrm{s}$ as shown in Fig. 11 . The test pipe has the diameter of $0.030 \mathrm{~m}$, submerged weight of $4.22 \mathrm{~N} / \mathrm{m}$. The constraint

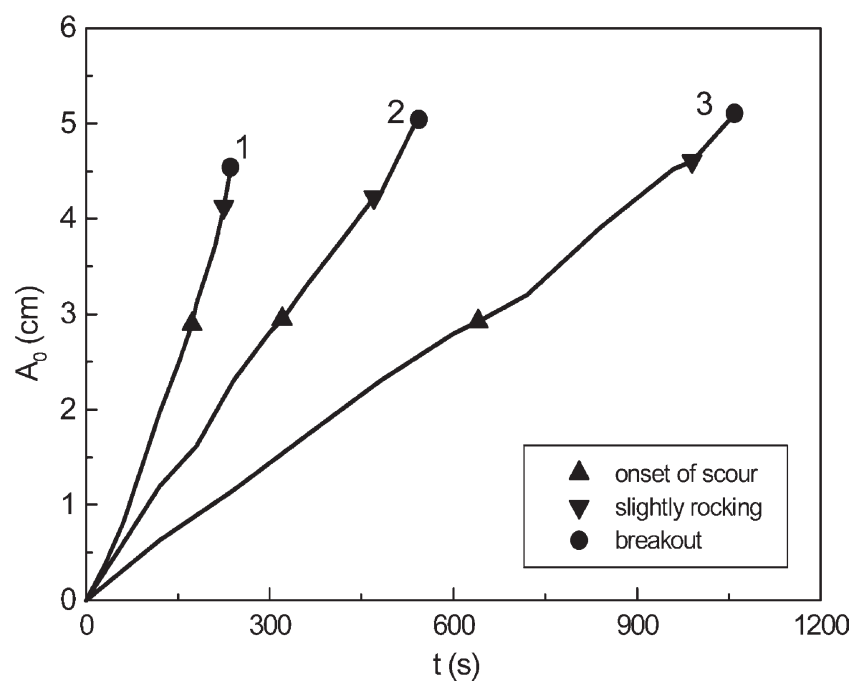

Fig. 11. Effects of loading velocity on pipe stability. 
condition is chosen as Case I. The test sand is a kind of medium sand, whose properties are listed in Table 1.

Fig. 11 shows that with the increase of $\dot{A}_{0}$, the oscillatory flow amplitude at which the pipe rocks slightly and the amplitude at which pipe loses stability increase, respectively, but the amplitude of oscillatory flow-induced sand scouring is affected slightly.

Experimental observation indicates that the smaller the $\dot{A}_{0}$, the higher the sand dune is formed beside the pipe, at the time when the pipe is losing stability. As analyzed in Section $2.3, \dot{A}_{0} \ll U_{\mathrm{m}}$, thus the change of $A_{0}$ is quasi-static at specific value of $U_{\mathrm{m}}$ (or $A_{0}$ ). Various $\dot{A}_{0}$ represents somehow the different oscillating times $(t / T)$ at the vicinity of certain value of $U_{\mathrm{m}}$. Therefore, the loading velocity (or the oscillating times) affects the sand scouring around pipe and eventually has influence on the stability of pipe.

\subsubsection{Effects of long-lasting oscillation at various amplitudes}

The storm growing is not always continuous, at different sea fields or various seasons at same sea field. Sometimes, long-lasting oscillation at various amplitudes occurs. To consider this situation, experiments with the following four types of loading history have been conducted, as shown in Fig. 12:

Type a: The amplitude increases at a constant velocity $\dot{A}_{0}=9.0 \times 10^{-3} \mathrm{~cm} / \mathrm{s}$.

Type b: The flow amplitude first increases at the former velocity till $A_{\mathrm{c}}=$ $2.60 \mathrm{~cm}$, then maintains at $A_{\mathrm{c}}$ for $5 \mathrm{~min}$, i.e. about 115 cycles, and finally increases as before.

Type c: Similar to Type b, except for $A_{\mathrm{c}}=4.15 \mathrm{~cm}$.

Type d: The flow amplitude first increases with the former velocity till $A_{\mathrm{c}}$ at which

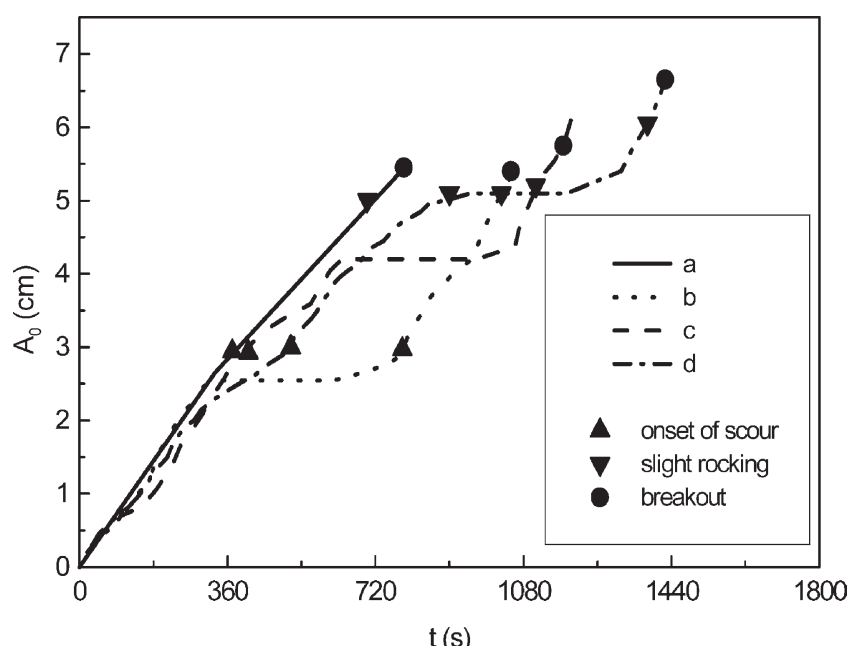

Fig. 12. Effects of long-lasting oscillating amplitude on the pipe stability. 
the pipe rocks slightly, then maintains at $A_{\mathrm{c}} 115$ cycles, and finally increases as before.

Fig. 12 indicates that when long-lasting oscillatory amplitude $A_{\mathrm{c}}$ is less than that of onset of scour $A_{\mathrm{s}}$ (Type b), it nearly does not have influence on the pipe stability. When $A_{\mathrm{c}}>A_{\mathrm{s}}$ (Types $\mathrm{c}, \mathrm{d}$ ), due to the effect of vortex, the sand grains pile up on both sides of the pipe, thereby the long-lasting oscillation increases the stability of pipeline. Furthermore, the pipe's slight rocking is not always followed by losing stability. If the flow amplitude does not rise after pipe begins to slightly rock, the pipe will return to the static condition again and more sediment is observed piling beside the pipe (Type d). After the flow amplitude increases to some higher level, the pipe slightly rocks again, and loses stability at higher flow amplitude.

All the types mentioned above imply that the wave-induced pipe instability is coupled with the sand scouring around pipe, and some intermittently growing storm could be beneficial for the pipe stability.

\section{Comparison with previous experiments}

The above experimental results show that, under the action of rapidly rising waveinduced loading, there exist different linear relationships between $F r$ and $G$ numbers for freely laid pipelines and anti-rolling pipelines, respectively. As to the medium sands, the least square fitting equations of the data in Figs. 8 and 10, can be given as

$$
\begin{aligned}
& \frac{U_{\mathrm{m}}}{(g D)^{1 / 2}} \\
& \quad=\left\{\begin{array}{l}
0.043+0.37 \frac{W_{\mathrm{s}}}{\gamma D^{2}}\left(0.18<\frac{W_{\mathrm{s}}}{\gamma D^{2}}<0.65\right) \text { freely laid pipes } \\
0.069+0.62 \frac{W_{\mathrm{s}}}{\gamma D^{2}}\left(0.18<\frac{W_{\mathrm{s}}}{\gamma D^{2}}<0.36\right) \text { anti-rolling pipes }
\end{array}\right.
\end{aligned}
$$

The equations give the relationships between water particle velocity, soil properties, pipe diameter and submerged weight of the pipe. All the parameters involved have obvious physical meaning. This line can be regarded as the critical line for pipe onbottom instability.

However, in the previous experiments (Allen et al., 1989; Brennodden et al., 1986; Wagner et al., 1987; Brennodden et al., 1989), mechanical actuator was used to simulate the real hydrodynamic forces upon pipelines. So the pipe-soil interaction models obtained by the experiments do not include wave parameters (see Eqs. (1), (2) and (3)). In order to compare with the previous experiment results, the calculation of hydrodynamic forces induced by waves on pipeline is essential. 


\subsection{Calculation of the wave-induced forces upon pipeline with Wake II model}

Historically, the wave-induced forces upon submarine pipeline used to be calculated with an adaptation of Morison's equation for both horizontal and vertical or a lift force taken to be proportional to the ambient velocity squared. However, it has been recognized that in the force model, the ambient velocity should be modified under the consideration of wake flow. Measurements showed that Morison's equation is lacking in its ability to predict the details in shape and magnitude of force time history.

Soedigdo et al. (1999) proposed a Wake II model, in which wake velocity correction was derived based on a closed-form solution to the linearized Navier-Stokes model for oscillatory flow and hydrodynamic forces' coefficients were determined based on start-up effects. Wake II model can be used for stability design calculations for pipelines on the seabed for regular waves without currents for various diameters. Sabag et al. (2000) pointed out that the Wake II model fits well with experiment results, and it is a great improvement on Morison's equation. The Wake II model is summarized in Appendix A.

Take the experiment on anti-rolling pipe with $W_{\mathrm{s}}=2.04 \mathrm{~N} / \mathrm{m}$ and $D=0.030 \mathrm{~m}$ as an example (see Figs. 13 and 14). Fig. 13 shows that the difference between the amplitude of $U_{\mathrm{e}}$ and that of $U(t)$ gets bigger with the increase of free stream velocity. The correction for wake velocity can significantly increase the effective velocity encountered by pipeline. The free stream, effective and wake velocities calculated with Wake II model are conceptually illustrated in Fig. 14. With Eqs. (24)-(27), the lift force and horizontal force can be calculated, as shown in Fig. 15.

Fig. 16 shows lift forces calculated with Wake II model and those predicted for the lift forces using Morison's equation. The predicted lift forces from Morison's equation are much smaller than that from Wake II model, since it reflects only the

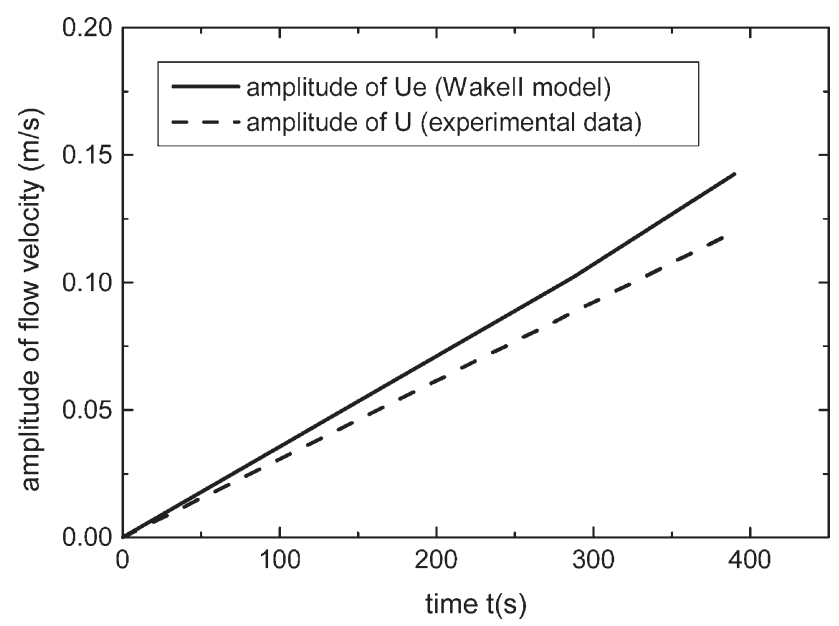

Fig. 13. The increasing of oscillatory flow amplitude $(D=0.030 \mathrm{~m})$. 


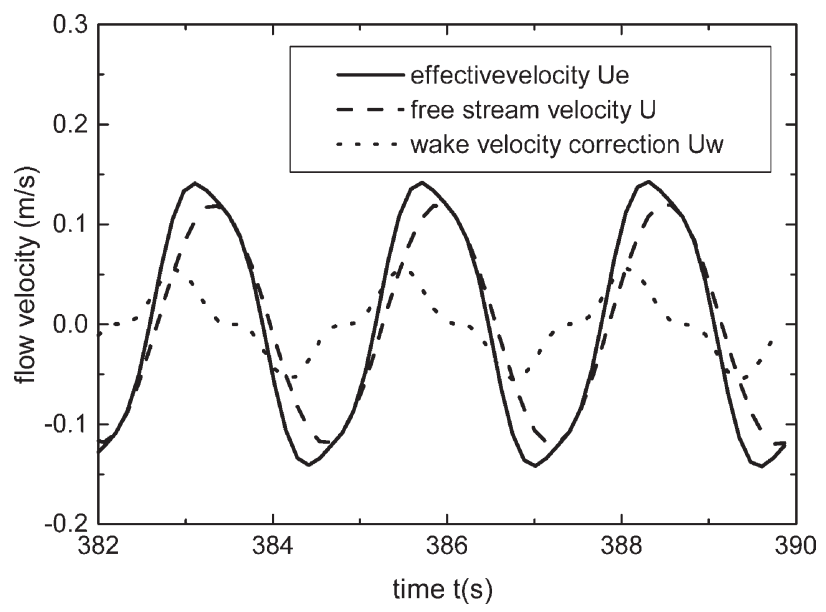

Fig. 14. The effective, free stream and wake velocities $(D=0.030 \mathrm{~m})$.

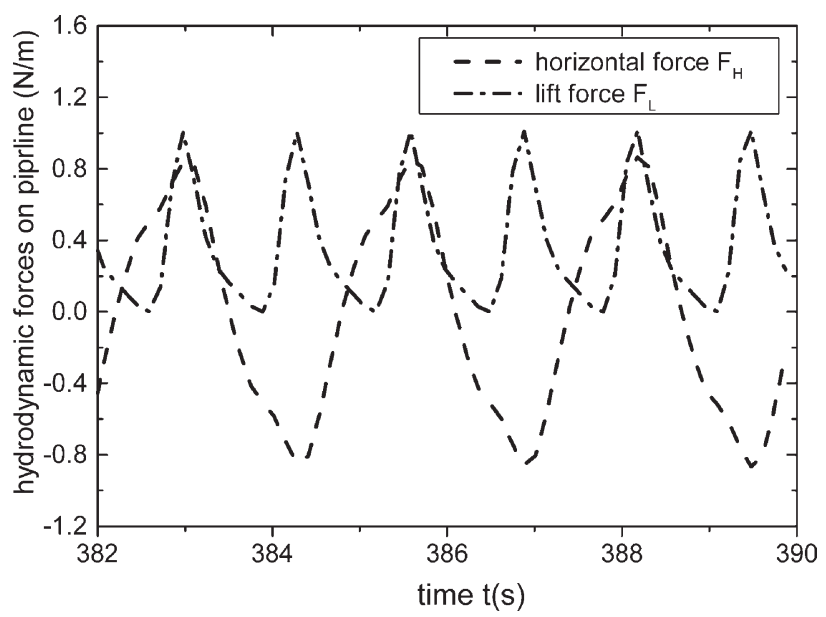

Fig. 15. Lift and horizontal forces on pipeline with Wake II $(D=0.030 \mathrm{~m})$.

ambient velocity magnitude. Typical horizontal force comparisons are shown in Fig. 17. The predicted horizontal forces from the Morison's equation are in close agreement with the horizontal forces predicted with Wake II model.

\subsection{Comparison with 'pipe-soil interaction' experiments}

As discussed in Section 4.1, under the rapidly increasing wave loading, the pipe suddenly moves away from its original site after a period of slight moving. During the breakout process, the pipeline must conquer the maximum soil resistance. The maximum coefficient $\mu$ of soil resistance can be expressed as 


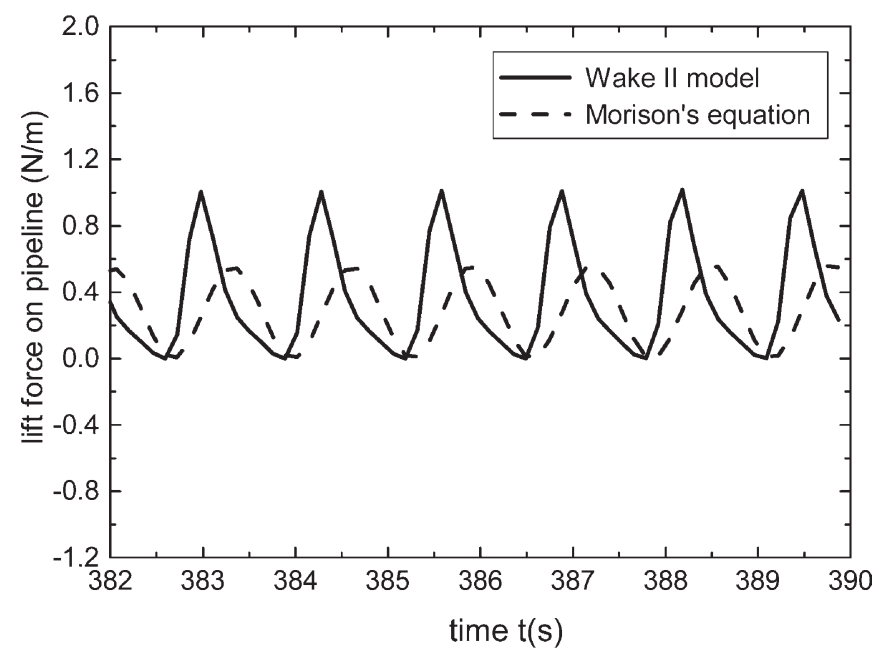

Fig. 16. Lift forces with Morison's equation and Wake II model $(D=0.030 \mathrm{~m})$.

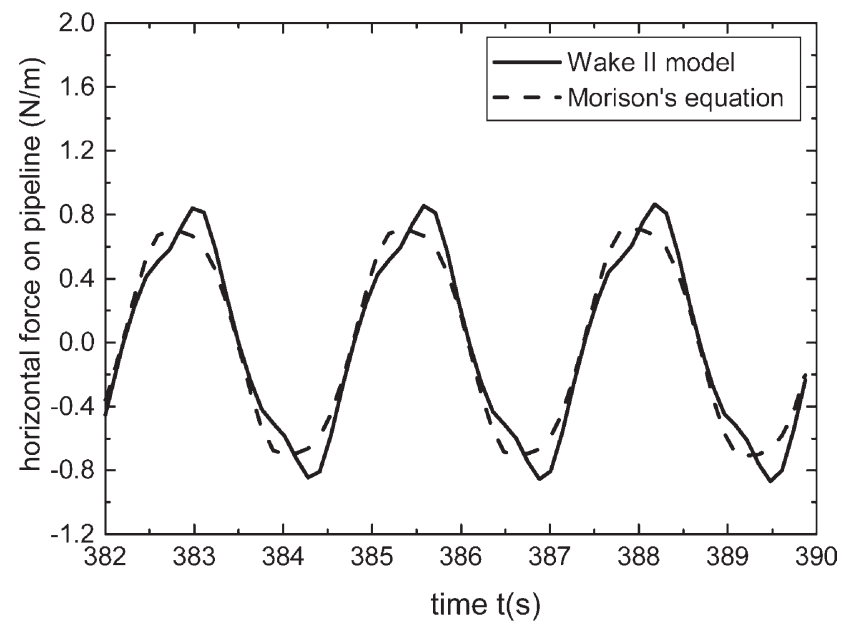

Fig. 17. Horizontal forces with Morison's equation and Wake II model $(D=0.030 \mathrm{~m})$.

$$
\mu=\left(\frac{F_{\mathrm{H}}(t)}{W_{\mathrm{s}}-F_{\mathrm{L}}(t)}\right)_{\max },
$$

where $F_{\mathrm{H}}(t)$ and $F_{\mathrm{L}}(t)$ are calculated with Eqs. (25) and (27), respectively. Thus, given the ambient velocities during the full period of pipe's losing stability, the maximum value of soil resistance can be obtained.

The maximum values of soil resistance for anti-rolling pipelines on medium sand 


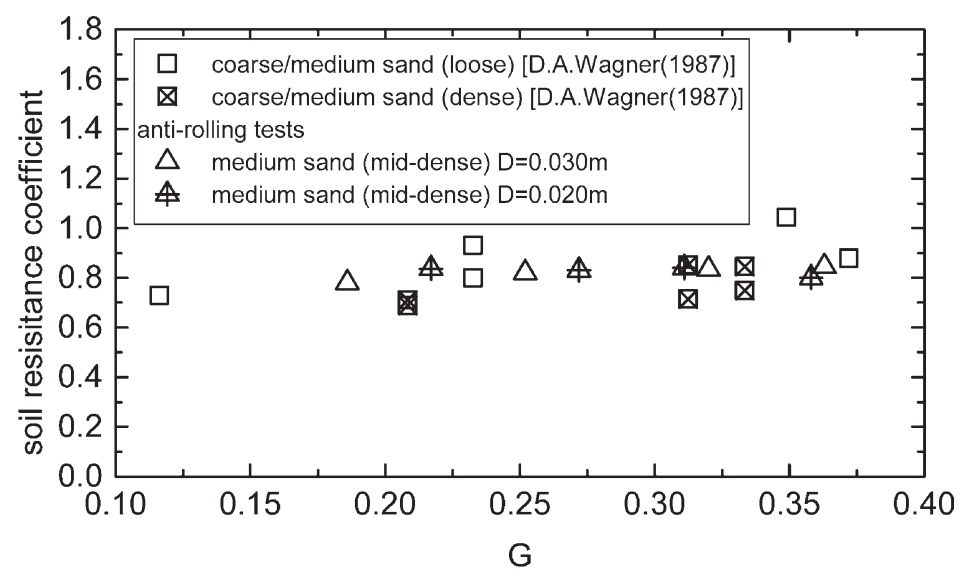

Fig. 18. Comparison with pipe-soil interaction experiment results of Wagner et al. (1987).

are shown in Fig. 18, together with that in previous pipe-soil interaction experiment results of Wagner et al. (1987). Typical test results of Brennodden et al. (1986) are given in Fig. 19. The average value of soil resistance in our experiments is about 0.83 , which is larger than the value obtained in previous pipe-soil interaction experiments. In the present experiments, the medium sand is moderate dense, whose relative density is 0.37 . Therefore, though our experiments are conducted with different loading style from previous pipe-soil interaction experiments, their results are comparable with the latter and more reasonable in the mechanism aspects for reflecting the coupling of wave-pipe-soil. Because of the insufficiency of the test data, the final model has not been obtained yet, but the relationships imply that they can serve as a supplementary analysis tool for pipeline stability.

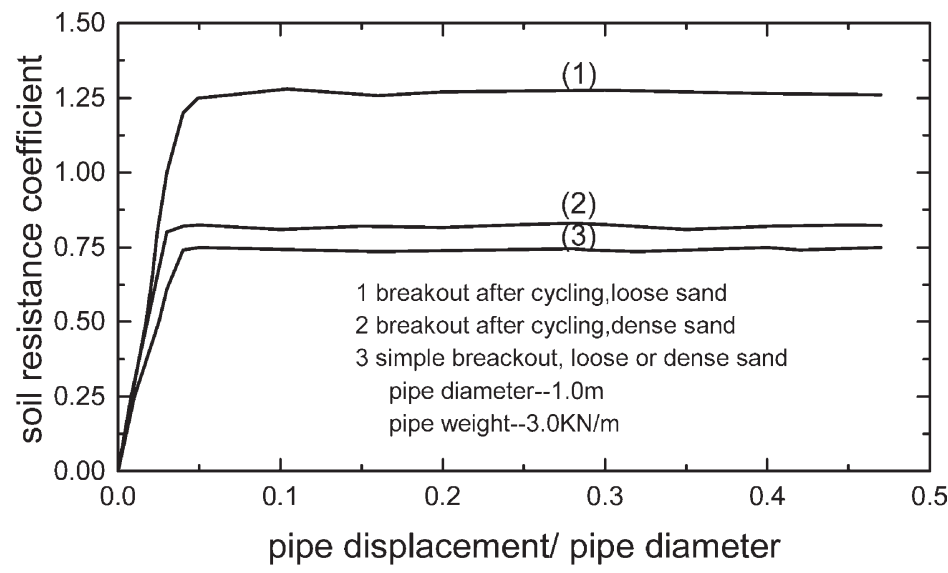

Fig. 19. Typical test results of Brennodden et al. (1986). 


\section{Conclusions}

Different from the previous experiments, U-shaped oscillatory flow tunnel is employed to investigate the wave-induced submarine pipeline instability in this paper. From the results presented above, the following conclusions can be made:

1. Froude number $(F r)$ and the non-dimensional pipe weight $(G)$ are two most important parameters in modeling wave-induced instability of untrenched pipeline. Based on the experimental results, different linear relationships between $F r$ and $G$ have been obtained for pipes with different restraint conditions, i.e. (a) freely laid pipelines and (b) anti-rolling pipelines. Moreover, three characteristic times in the process of the pipe's losing stability are revealed.

2. Based on Wake II model, the current wave-soil-pipe interaction test results and the results of previous pipe-soil interaction tests are compared. It is indicated that the results of the two types of tests are comparable. The obtained $F r-G$ relationships can be used for supplementary analysis of criterion for pipeline instability in design procedure.

3. In consideration of the actual field conditions, different loading histories are used to explore the effects of them on the pipeline instability. It is found that the scouring of sand beside the pipeline is the main result of the different loading histories, and affects the pipeline stability eventually.

4. Sand scour, as an indicator of the wave-soil-pipe coupling, is detected in our experiments. But, in the previous experiments with actuator, it could not be modeled. Therefore, the current hydrodynamic experiments are more reasonable in the mechanism aspects, which reflect the wave-soil-pipeline coupling effects.

\section{Acknowledgements}

This study was jointly supported by the Chinese National Scientific Foundation Projects (19772057) and the key project of the Ninth 5-year Plan of Chinese Academy of Sciences (KZ951-A1-405-01). This work was also supported by ARC Small Grant (2001) at Griffith University.

\section{Appendix A. Wake II model}

Wake II model proposed by Soedigdo et al. (1999) is a hydrodynamic force model for prediction of forces on pipelines. The wake and start-up effects are considered in the model.

The wake velocity correction is corrected by using a closed-form solution to the linearized Navier-Stokes equations for oscillatory flow. By assuming that the eddy 
viscosity in the wake is only time dependent and of harmonic sinusoidal form, the wake velocity correction affecting pipe in periodic flow can be derived as

$$
U_{\mathrm{w}}(t)=\frac{(\pi)^{1 / 2} \operatorname{erf}\left(\frac{1}{2} C_{2} \sin ^{n}(\omega t+\phi)\right) U_{\mathrm{m}} C_{1}}{C_{2}},
$$

where $C_{1}, C_{2}, \phi$ and $n$ are empirical parameters relative to current $\mathrm{KC}$ number, as shown in Fig. 20. The effective velocity $U_{\mathrm{e}}$ can be determined as the sum of the free stream velocity $U(t)$ and the wake velocity correction $U_{\mathrm{w}}(t)$ as

$$
U_{\mathrm{e}}(t)=U(t)+U_{\mathrm{w}}(t)
$$

When the effective velocity is known, the force model expressions for the drag, lift and inertial forces are:

Drag force: $F_{\mathrm{D}}=0.5 \rho_{\mathrm{w}} D C_{\mathrm{D}}(t)\left|U_{\mathrm{e}}\right| U_{\mathrm{e}}$,

Lift force: $F_{\mathrm{L}}=0.5 \rho_{\mathrm{w}} D C_{\mathrm{L}}(t) U_{\mathrm{e}}^{2}$,

Inertia force: $F_{\mathrm{I}}=\frac{\pi D^{2}}{4} \rho_{\mathrm{w}}\left[C_{\mathrm{M}} \frac{\mathrm{d} U}{\mathrm{~d} t}-C_{\mathrm{Aw}} \frac{\mathrm{d} U_{\mathrm{w}}}{\mathrm{d} t}\right]$.
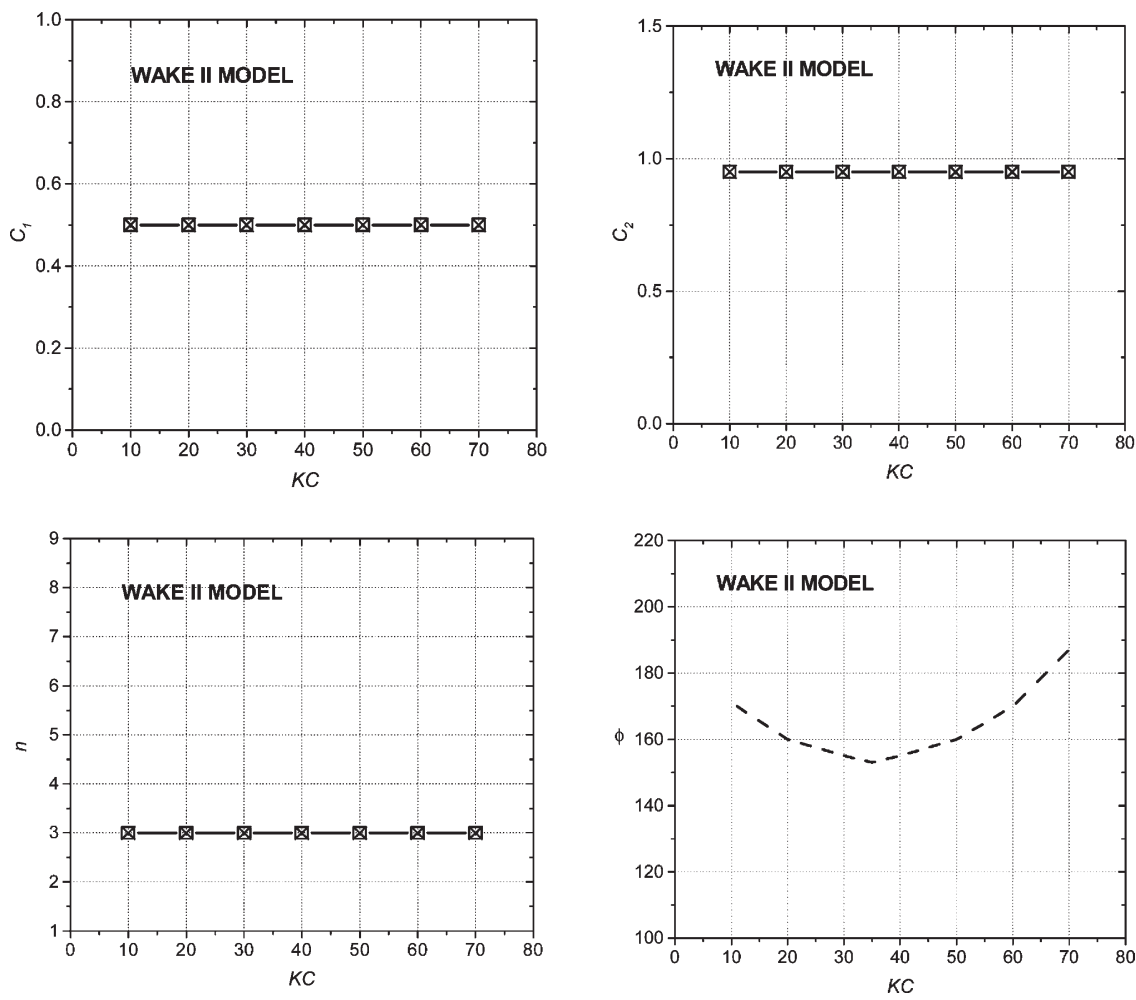

Fig. 20. Effect of KC on parameters $C_{1}, C_{2}, \phi$ and $n$ (Soedigdo et al., 1999). 
The horizontal hydraulic force is the sum of drag force and inertia force

$$
F_{\mathrm{H}}=F_{\mathrm{D}}+F_{\mathrm{I}} \text {, }
$$

where $C_{\mathrm{M}}$ is the inertia coefficient for ambient velocity, $C_{\mathrm{M}}=2.5 ; C_{\mathrm{AM}}$ the added mass coefficient associated with the wake flow passing the pipe, $C_{\mathrm{AM}}=0.25$; and $C_{\mathrm{D}}(t), C_{\mathrm{L}}(t)$ are time dependent drag and lift coefficients, respectively and can be determined by the so-called 'start-up' function, which is relative to the distance the water particle travels after a zero crossing in the total effective velocity.

\section{References}

Allen, D.W., Lammert, W.F., Hale, J.R., Jacobsen, V., 1989. Submarine pipeline on-bottom stability: recent AGA research. Proc. Offshore Technol. Conf., OTC 6055, 121-132.

Brennodden, H., Lieng, J.T., Sotberg, T., Verley, R.L.P., 1989. An energy-based pipe-soil interaction model. Proc. Offshore Technol. Conf., OTC 6057, 147-158.

Brennodden, H., Sveggen, O., Wagner, D.A., Murff, J.D., 1986. Full-scale pipe-soil interaction tests Proc. Offshore Technol. Conf., OTC 5338, 433-440.

Chakrabarti, K., 1994. Offshore Structure Modeling. JBW Printers \& Binder Pte Ltd, Southampton, Boston.

Chiew, Y.M., 1990. Mechanics of local scour around submarine pipelines. J. Hydraulic Eng. 116, 515529.

Det norske Veritas, 1988. On-bottom stability design of submarine pipelines, Recommended Practice E305.

Hale, J.R., Lammert, W.F., Allen, D.W., 1991. Pipeline on-bottom stability calculations: comparison of two state-of-the-art methods and pipe-soil model verification. Proc. Offshore Technol. Conf., OTC 6761, 567-581.

Herbich, J.B., 1985. Hydromechanics of submarine pipelines: design problems. Can. J. Civil Eng. 12, 863-874.

Lyons, C.G., 1973. Soil resistance to lateral sliding of marine pipelines. Proc. Offshore Technol. Conf., OTC 1876, 479-484.

Mao, Y., 1988. Seabed scours under pipelines. In: Proceedings of the Seventh International Symposium on Offshore Mechanics and Arctic Engineering (OMAE), pp. 33-38.

Poorooshasb, F., 1990. On centrifuge use for ocean research. Mar. Geotechnol. 9, 141-158.

Sabag, S.R., Edge, B.L., Soedigdo, I.R., 2000. Wake II model for hydrodynamic forces on marine pipelines including waves and currents. Ocean Eng. 27, 1295-1319.

Soedigdo, I.R., Lambrakos, K.F., Edge, B.L., 1999. Predicton of hydrodynamic forces on submarine pipelines using an improved wake II model. Ocean Eng. 26, 431-462.

Sumer, B.M., Fredsoe, J., 1991. Onset of scour below a pipeline exposed to waves. Int. J. Offshore Polar Eng. 1, 189-194.

Wagner, D.A., Murff, J.D., Brennodden, H., Sveggen, O., 1987. Pipe-soil interaction model. Proc. Offshore Technol. Conf., OTC 5504, 181-190. 